\title{
Combination of Statins and NSAIDs as a Potential Novel Addition to the Limited Anti-Breast Cancer Weaponry: A True Synergistic Model
}

\author{
Asian Pac J Cancer Prev, 20 (5), 1295-1296
}

\section{Dear Editor}

One of the most important challenges of today's breast cancer therapy is the lack of response to treatment and the recurrence of tumor after seemingly successful and complete remissions. Many clinical and preclinical studies that have been conducted to achieve a correct model for predicting and acquiring the right prognosis for these patients haven't reached the desired outcome (Ahmad, 2013). Although we haven't truly figured out the underlying mechanisms and effecting factors in molecular pathways involved in cancer and the hierarchy of events that predispose a patient to recurrence of tumor but several newer studies have indicated that the following pathways are specially involved in tumor relapse: the hormonal status of tumor (ER, PR), her2, cancer stem cell, RhoA protein expression, increased phosphatase and tensin homolog (PTEN) activity and suppression of apoptosis mechanisms(Ahmad, 2013; Zhang et al., 2013).

Despite recent advances in diagnosis and treatment of breast cancer, unfortunately, in many cases, we are still unable to provide an adequate treatment and survival for the patients. So, to overcome these problems there is a need for more investigational studies and usage of newer and more experimental modalities. Recently meta-analysis and systematic reviews have shown that older agents such as statins and NSAIDs might have a role in breast cancer treatment. Clinical evidence of above-mentioned studies is pretty exciting for decreasing relapse, mortality and increasing of patient's quality of life (Huang et al., 2015; Van Wyhe et al., 2017). These two drugs are prescribed together in many cardiovascular disorders. Some hypothesize that these two classes of drugs have synergistic effects. Regards to probable synergistic effects of these drugs and also the possibility to reduce the dosage which in turn will result in decreased side effects and better tolerance of them (Jalving et al., 2005). Authors suggest combination therapy with statins and NSAIDs as a potential novel adjunctive treatment for breast cancer. In the following paragraphs, we will briefly explain the scientific basis for our claim.

Many studies (including in vitro and in vivo studies) have shown a link with the usage of statins and NSAIDs in different cancers and lower rates of mortality (Xiao and Yang, 2008). Also, studies on colon cancer cell lines have demonstrated that this combination therapy when applied as an adjunctive to conventional therapies, results in a better rate of apoptosis and cell cycle arrest at G0/ G1 Phase (more than any other monotherapy) (Xiao et al., 2008). Also this combination therapy results in better inhibition of RhoA pathway as an important factor in tumor aggression and metastasis by negative regulation and utilization on both p21Cip1/Waf1 and p27Kip1 and increase in the levels of these $2 \mathrm{CDK}$ inhibitors and also increase in the level of membrane bound RhoB (as an possible antitumor agent) in contrast to RhoA and increased PTEN activity (Adnane et al., 1998). Indeed NSAIDs when used with statins via abolishing phosphorylation of Akt have a far better chance of inhibiting the Akt pathway (as an important cellular apoptosis pathway) than any of them used alone (Xiao et al., 2008).

Regarding the above-mentioned studies and the important role of these pathways in breast cancer, we suggest the design of in vitro studies and double-blinded randomized control trials to better show the effect of these drugs and to the extent in which they can be helpful in our ever demanding fight against cancer.

Keywords: Statins- NSAIDs- Breast cancer-combination treatment

\section{Conclusion}

Not applicable.

\section{Declarations}

Ethics approval and consent to participate: Not applicable.

\section{Consent for publication}

Not applicable.

\section{Availability of data and material}

Not applicable.

Competing interests

None declared.

Funding

None.

Conflict of interest

None.

Asian Pacific Journal of Cancer Prevention, Vol 20 


\section{Acknowledgements}

We thank Mohammad Panahi for his undeserved help.

\section{References}

Adnane J, Bizouarn FA, Qian Y, Hamilton AD, Sebti SM (1998). p21(WAF1/CIP1) is upregulated by the geranylgeranyltransferase I inhibitor GGTI-298 through a transforming growth factor beta- and Sp1-responsive element: involvement of the small GTPase rhoA. Mol Cell Biol, 18, 6962-70.

Ahmad A (2013). Pathways to breast cancer recurrence. ISRN Oncol, 2013.

Bellizzi A, Mangia A, Chiriatti A, et al (2008). RhoA protein expression in primary breast cancers and matched lymphocytes is associated with progression of the disease. Int J Mol Med, 22, 25-31.

Huang M, Prendergast GC (2006). RhoB in cancer suppression. Histol Histopathol, 21, 213-8.

Huang XZ, Gao P, Sun JX, et al (2015). Aspirin and nonsteroidal anti-inflammatory drugs after but not before diagnosis are associated with improved breast cancer survival: a meta-analysis. Cancer Causes Control, 26, 589-600.

Jalving M, Koornstra JJ, De Jong S, De Vries EG, Kleibeuker JH (2005). Review article: the potential of combinational regimen with non-steroidal anti-inflammatory drugs in the chemoprevention of colorectal cancer. Aliment Pharmacol Ther, 21, 321-9.

Van Wyhe RD, Rahal OM, Woodward WA (2017). Effect of statins on breast cancer recurrence and mortality: a review. Breast Cancer Targets Therapy, 9, 559.

Xiao H, Yang CS (2008). Combination regimen with statins and NSAIDs: a promising strategy for cancer chemoprevention. Int J Cancer, 123, 983-90.

Xiao H, Zhang Q, Lin Y, Reddy BS, Yang CS (2008). Combination of atorvastatin and celecoxib synergistically induces cell cycle arrest and apoptosis in colon cancer cells. Int J Cancer, 2008.

Zhang HY, Liang F, Jia ZL, Song ST, Jiang ZF (2013). PTEN mutation, methylation and expression in breast cancer patients. Oncol Lett, 6, 161-8.

\section{Abdolhassan Talaiezadeh ${ }^{1}$, Amir Mohammad Papan ${ }^{1}$, Pedram Nazari ${ }^{1 *}$, Seyedeh Parvin Mousavi Ghanavati ${ }^{1}$, Ali Ramazani ${ }^{2}$}

${ }^{1}$ Cancer Research Center, ${ }^{2}$ Department of Virology, Ahvaz Jundishapur University of Medical Sciences, Ahvaz, Iran. *For Correspondence: dr.pedramnazari@gmail.com 